\title{
Layout Optimization of Two Autonomous Underwater Vehicles for Drag Reduction with a Combined CFD and Neural Network Method
}

\author{
Wenlong Tian, ${ }^{1,2}$ Zhaoyong Mao, ${ }^{1,2}$ Fuliang Zhao, ${ }^{1}$ and Zhicao Zhao ${ }^{3}$ \\ ${ }^{1}$ School of Marine Science and Technology, Northwestern Polytechnical University, Xian 710072, China \\ ${ }^{2}$ Key Laboratory for Unmanned Underwater Vehicle, Northwestern Polytechnical University, Xian 710072, China \\ ${ }^{3}$ Xian Institute of Applied Optics, Xian 710065, China
}

Correspondence should be addressed to Wenlong Tian; tianwenlong@nwpu.edu.cn

Received 8 August 2017; Accepted 12 November 2017; Published 7 December 2017

Academic Editor: Junpei Zhong

Copyright (C) 2017 Wenlong Tian et al. This is an open access article distributed under the Creative Commons Attribution License, which permits unrestricted use, distribution, and reproduction in any medium, provided the original work is properly cited.

This paper presents an optimization method for the design of the layout of an autonomous underwater vehicles (AUV) fleet to minimize the drag force. The layout of the AUV fleet is defined by two nondimensional parameters. Firstly, three-dimensional computational fluid dynamics (CFD) simulations are performed on the fleets with different layout parameters and detailed information on the hydrodynamic forces and flow structures around the AUVs is obtained. Then, based on the CFD data, a backpropagation neural network (BPNN) method is used to describe the relationship between the layout parameters and the drag of the fleet. Finally, a genetic algorithm (GA) is chosen to obtain the optimal layout parameters which correspond to the minimum drag. The optimization results show that (1) the total drag of the AUV fleet can be reduced by $12 \%$ when the follower AUV is located directly behind the leader AUV and (2) the drag of the follower AUV can be reduced by $66 \%$ when it is by the side of the leader AUV.

\section{Introduction}

Autonomous underwater vehicles (AUVs) are a kind of self-sailing, self-executing underwater robots, which play an important role in expanding people's knowledge of the ocean. AUVs are capable of a wide range of applications, such as pipeline inspection [1,2], underwater search and rescue, mine-sweeping [3], and oceanographic exploration [4]. The tasks of current AUVs are relatively single, and in the future, AUVs will be able to perform a variety of tasks. Therefore, AUVs will be composed of more complex structures and control systems, as well as more sensors and functional modules. Another solution for complex tasks is to use a fleet of simple AUVs, among which each one has a specific function, so that the fleet can complete complex tasks with simple AUVs and at a lower cost.

When the AUVs travel in a fleet, the layout of the fleet becomes a key problem. Observations of animal motions such as birds in flocks suggest that some energy benefit may be obtained by certain fleet shapes [5]. It is known to all that migrating geese fly in a $\mathrm{V}$ or I formation for long distance migrations (Figure 1). The leader goose produces high speed updrafts in wake, which help the follower geese to save energy. Similarly, the leader AUV in the fleet produces low speed wake and low pressure side flows. Therefore, the follower AUVs could possibly use the velocity difference or pressure difference to minimize the drag and save energy.

The drag of an individual AUV can be reduced by optimizing the shape of the hull, propeller, and surface control $[6,7]$. Alvarez et al. introduced an optimization method for the shape optimization of an AUV moving near the water surface [8]. They used a panel model to predict the wave resistance of a revolution body and a simulated annealing algorithm to optimize the geometric parameters that minimize the wave resistance. Kim et al. proposed a Computational Fluid Dynamics (CFD) method for the optimization of monohull ships with the minimum drag [9]. Joung et al. proposed a procedure using the CFD method 


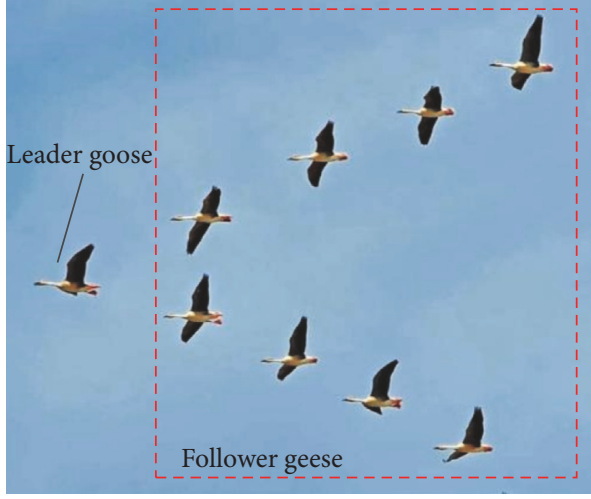

(a)

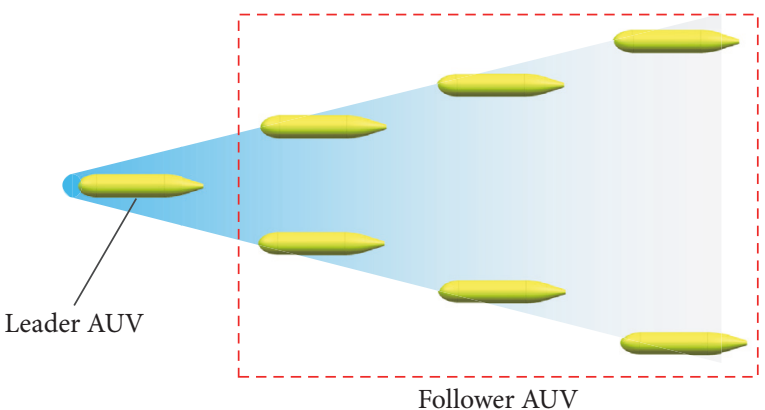

(b)

FIGURE 1: (a) Migrating geese and (b) possible layout of an AUV fleet.

and a Design of Experiments (DOE) optimization for the hull optimization [10]. Sun et al. established an energy consumption model for an underwater glider and optimized the shape of the glider with a combined CFD and Efficient Global Optimization (EGO) method [11].

CFD simulations can predict the hydrodynamic performance of the AUV with high accuracy, however, at the cost of time. Combined optimization method based on the CFD, Back-Propagation Neural Network (BPNN), and Genetic Algorithm (GA) can efficiently save the simulation time to obtain the optimal target. BPNN and GA are used at some of the nonlinear data to find the optimal value $[12,13]$. Safikhani et al. studied the multiobjective optimization of nanofluid flow in flat tubs, which combined with a CFD, artificial neural networks, and genetic algorithms [14]. They got important design information about nanofluids and flat tubes. Avc1 et al. studied the optimization of the deign parameters of a home refrigerator using CFD and artificial neural network [15]; they showed that CFD simulation and the ANN can determine the best value for the refrigerator design.

Inspired by the above research, this paper introduces an optimization procedure of an AUV fleet, which contains two AUVs, to minimize its drag. The layout of the fleet is defined by two nondimensional parameters. Three-dimensional CFD simulations are performed to find the drag of each case. Then BPNN and GA optimization method is used to find the optimal layout which has the minimum drag.

\section{Geometry Configuration}

2.1. The AUV Hull Design. The AUV considered in this paper has a torpedo-like axisymmetric shape, which is characterized with a length of $L=1.3 \mathrm{~m}$ and a maximum diameter of $D=0.2 \mathrm{~m}$, resulting in a slenderness ratio (the ratio of the length to the diameter) of 6.5. The two-dimensional sketch of the AUV is shown in Figure 2. The designed AUV is composed of a nose section, a middle section, and a tail section. The lengths of the three sections are $0.2 \mathrm{~m}, 0.7 \mathrm{~m}$, and $0.4 \mathrm{~m}$, respectively. In order to obtain a low-drag shape, the Myring Equation [17] is used to design the shape of the AUV nose. The Myring Equation is widely used in the
TABLE 1: Dimensional parameters of the AUV.

\begin{tabular}{lcccccc}
\hline$D(\mathrm{~m})$ & $D_{t}(\mathrm{~m})$ & $L(\mathrm{~m})$ & $L_{h}(\mathrm{~m})$ & $L_{c}(\mathrm{~m})$ & $L_{t}(\mathrm{~m})$ & $\theta\left(^{\circ}\right)$ \\
\hline 0.20 & 0.04 & 1.30 & 0.20 & 0.70 & 0.40 & 30 \\
\hline
\end{tabular}

design of AUV hulls [10]. The expression for the nose shape is determined by

$$
r(x)=\frac{1}{2} D\left[1-\left(\frac{x-L_{h}}{x}\right)^{2}\right]^{1 / 1.8}
$$

where $x$ is the position along the rotation axis, $r$ is the radius at a specific $x$, and $L_{h}$ is the length of the nose. The tail section is smoothly transited from the mid-section to a circular cone, which has a cone angle of $30^{\circ}$. The diameter of the rear surface is $0.04 \mathrm{~m}$. Table 1 shows the values of the dimensional parameters.

2.2. Parameters of the AUV Fleet. As shown in Figure 3, a coordinate frame is assigned to the nose of the leader AUV, with its horizontal axis pointing right and its vertical axis pointing to the side of the follower AUV. The layout of the AUV fleet can be characterized by two parameters, $a$ and $b$, which represent the relative location of two adjacent AUVs in the fleet. To eliminate the dimensional effects, $a$ and $b$ are normalized by dividing the length of the AUV,

$$
\begin{aligned}
& a=\frac{y_{f}}{L}, \\
& b=\frac{x_{f}}{L},
\end{aligned}
$$

where $x_{f}$ and $y_{f}$ are the position of the follower AUV in the proposed coordinate frame.

A series of fleet layouts can be described by changing the two design parameters, $a$ and $b$. Before the optimization procedure, 53 groups of CFD simulations are performed as sample points with $a$ varying from 0.00 to 0.60 and $b$ varying from 0.00 to 2.00. It should be noted that simulations for $a \leq$ 0.2 and $b \leq 1.00$ are not performed because of the geometric interference between the two AUVs in these situations. 


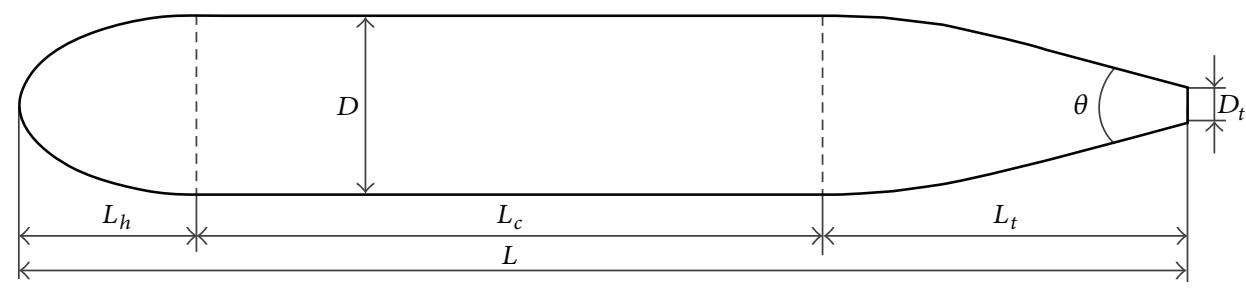

Figure 2: Shape of the AUV.

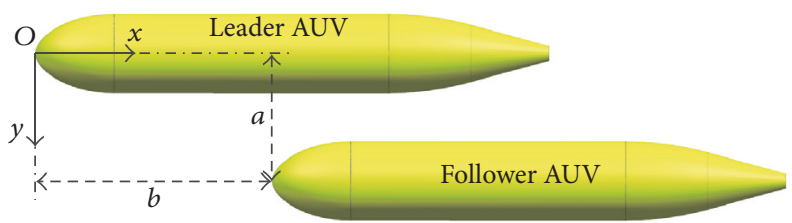

Figure 3: Parameter definition for the AUV fleet.

This study aims optimize the drag of the AUV fleet. The drag is normalized in the following way:

$$
\begin{aligned}
C_{d l} & =\frac{F_{x l}}{(1 / 2) \rho S U^{2}}, \\
C_{d f} & =\frac{F_{x f}}{(1 / 2) \rho S U^{2}}, \\
C_{d \text { sum }} & =C_{d l}+C_{d f},
\end{aligned}
$$

where $C_{d l}, C_{d f}$, and $C_{d \text { sum }}$ are the drag coefficient of the leader $\mathrm{AUV}$, the follower AUV, and the fleet, respectively, $\rho$ is the water density and is equal to $998 \mathrm{~kg} / \mathrm{m}^{3}, S$ is the area of the cross-section of the AUV, and $U$ is the velocity of the AUV.

\section{CFD Method}

The drag coefficients and the flow structures for each simulation case are obtained by the CFD approaches separately. A group of transient representations of the three-dimensional flow are carried out to improve the efficiency of the optimization process. Further, the results of this numerical model will be compared with existing experimental data for the model validation, which will be discussed in Section 3.4.

3.1. Governing Equations. The prediction of the flow around the AUVs is based on the incompressible Navier-Stokes equations [18],

$$
\begin{gathered}
\frac{\partial \rho}{\partial t}+\nabla \rho \vec{v}=0 \\
\frac{\partial}{\partial t}(\rho \vec{v})+\nabla(\overrightarrow{\rho v} \vec{v})=-\nabla p+\nabla \overline{\bar{\tau}}
\end{gathered}
$$

where $t$ represents time, $\vec{v}$ is the vector of velocity, $p$ is pressure, and $\overline{\bar{\tau}}$ refers to the stress tensor.

The shear stress transport (SST) $k$ - $\omega$ turbulence model was selected to model the turbulence terms of the RANS equations [18]. The SST $k$ - $\omega$ turbulence model is able to model the transport of turbulent shear stress and gives accurate predictions on the onset and amount of flow separation under adverse pressure gradients and has been successfully used in the CFD simulation of complex flows [11, 19-22].

3.2. Computational Domain and Boundary Conditions. The computational domain is a finite space used to simulate the flow around the AUV. In order to minimize the effect of block caused by the AUV, a rectangular domain with a dimension of $8 L \times 20 D \times 15 D$ is selected, resulting in a maximum block ratio of $0.67 \%$, as shown in Figure 4 . The length of the domain is $8 \mathrm{~L}$ and the leader AUV is placed in the mid-plane of the domain and $3 L$ to the left boundary. The follower AUV is in the same horizontal plane with the leader AUV and is offset in the coordinate frame.

Steady and uniform velocity inlet boundary is set at the left surface of the domain. The magnitude of the inflow velocity is $6 \mathrm{~m} / \mathrm{s}$ and a moderate turbulence intensity of $5 \%$ is chosen. Pressure outlet boundary is chosen for the right surface of the domain, which has the same turbulence intensity with the inlet boundary. Smooth wall conditions are imposed at the four-side surfaces, where the shear effects are neglected to minimize the influences of the walls. Standard wall conditions are applied to the surfaces of both AUVs.

3.3. Mesh Generation. In order to obtain more accurate results with smaller number of grids, the computational domain is meshed with structured hexahedral grids, as shown in Figure 5. Because the flow around the AUVs is more complex and has higher velocity gradients, a higher density of grid is set in this place to capture the development of flow with accuracy. Particularly, prism layer grid elements (Figure 5(c)) are generated from the wall surfaces to refine the mesh quality near the boundary layer flows. The initial height of the prism layer above the wall surfaces is an important indicator for the quality of the mesh. In this study, we use the nondimensional $y^{+}$value to evaluate the quality of the prism layer and a maximum value of 1 is chosen for all meshes. For a smoother 


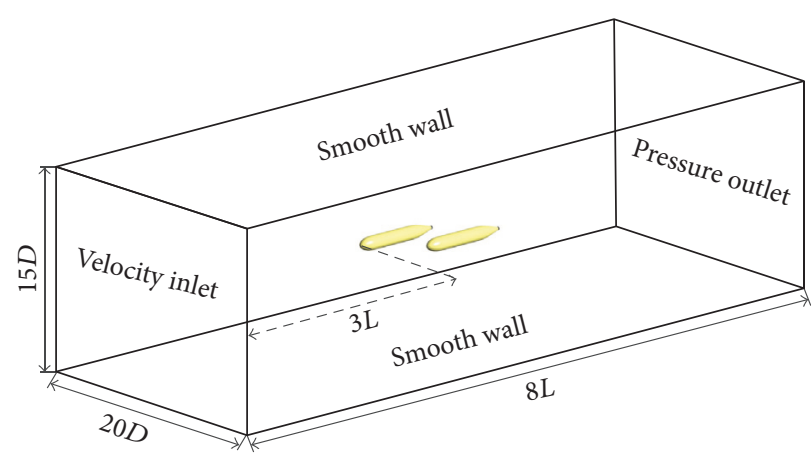

FIGURE 4: Computational domain and boundary conditions.

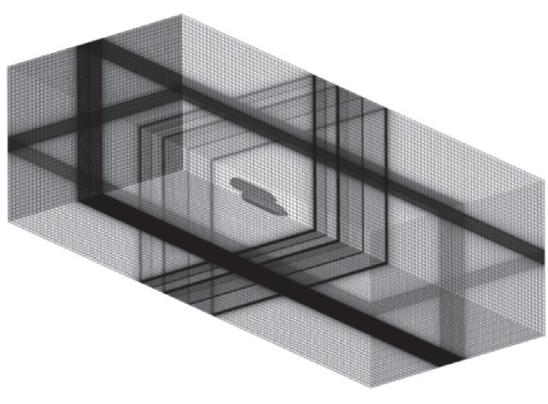

(a)

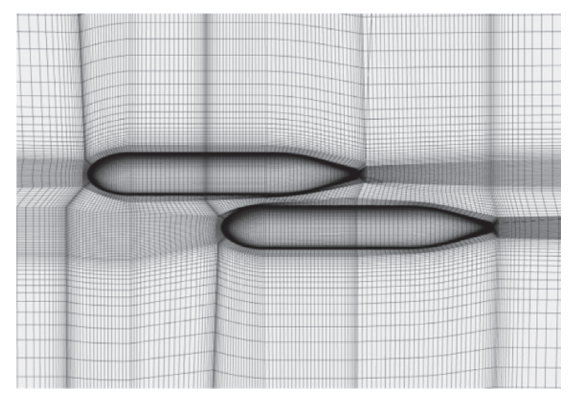

(b)

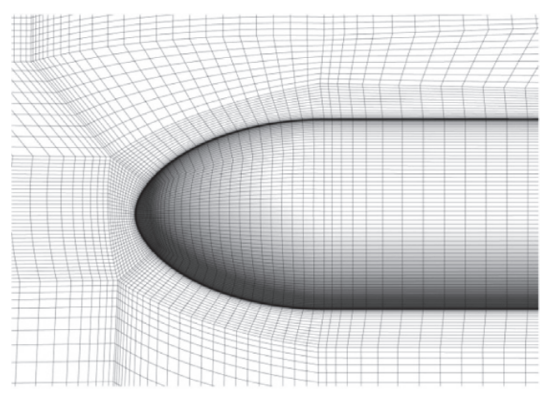

(c)

FIGURE 5: Details of the computation mesh: (a) overview; (b) grids around the vehicle; and (c) prism layer grid elements.

TABLE 2: Results of the mesh verification study.

\begin{tabular}{lcc}
\hline Mesh & Grid number & $C_{d}$ \\
\hline Coarse & 1670000 & 0.0786 \\
Mediate & 2800000 & 0.0774 \\
Fine & 3760000 & 0.0771 \\
\hline
\end{tabular}

transition of the adjacent elements, a general mesh growth rate of 1.2 is chosen for all grids.

3.4. Verification and Validation. A mesh verification study is carried out to determine the proper density of mesh. The verification simulations are performed on a single AUV with different meshes. Three meshes, with approximately 3.76 million (fine mesh), 2.80 million (mediate mesh), and 1.67 million (coarse mesh) elements, respectively, are generated for the grid resolution verification. The drag coefficients obtained by the three meshes are listed in Table 2. It is found that the coarse mesh predicts slightly higher $C_{d}$ and the other two give almost the same results. Therefore, the mediate mesh is chosen for the following simulations. It should be noted that, for the simulations of the AUV fleet, the grid density is kept the same with that of the single AUV and the number of elements is about 5.5 million due to the increased number of AUVs.

A validation study is performed for the validation of the proposed numerical model. Simulations are performed according to an experiment of twin parallel bare prolate spheroids with transverse separations [16] ( $a$ varies and $b=$ 0 in current study), as shown in Figure 6. The length of the model was $1.2 \mathrm{~m}$ and the maximum diameter was $0.2 \mathrm{~m}$, which is very close to the size of the AUV in this study. The testing inlet velocity was $40 \mathrm{~m} / \mathrm{s}$, corresponding to a Reynolds number of $3.2 \times 10^{6}$ according to the length of the model. This Reynolds number was also close to the current simulation. The calculated coefficients of drag and side force of the top spheroid are then compared with those obtained by experiment [16] and a previous CFD study [23] (Figure 7). It is noticed that the current CFD model provides more accurate results than that presented by Molland and Utama [23]. The maximum relative errors of the predicted drag and side force are $3.3 \%$ and $9.1 \%$, respectively. This proves that the CFD model in this study can predict the forces of two AUVs with acceptable accuracy.

\section{Optimal Design Method}

This paper establishes an optimization method based on the combination of CFD method, BPNN and GA. The drag coefficients and the flow structures for 53 different layouts are obtained by the CFD approaches separately. The results are then used to build the agent model between $C_{d}$ and the design parameters ( $a$ and $b$ ) using BPNN. Finally, GA is applied to search for the optimal $C_{d}$ together with the values for the corresponding design parameters based on the agent model.

4.1. BP Neural Network. The artificial neural network is widely accepted as an alternative to providing solutions to 


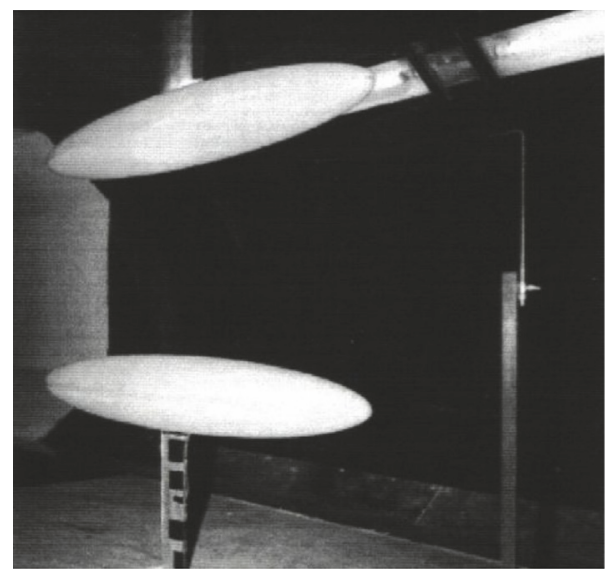

FIgURE 6: Wind tunnel test of the twin spheroids [16].

complex and ambiguous problems [24, 25]. In this study, a back-propagation algorithm that optimizes weighted connections by allowing errors to propagate from the output layer to the input layer is used to train the network. The topology of the BPNN in present study is shown in Figure 8. The structure has two input layers, five hidden layers and one output layer. Each node of the structure represents a neuron, and the nodes between the layers and the layers are connected by weights.

The BPNN is working based on the following principle:

(1) According to CFD simulation results, determine the network input data and output data $(X, Y) . H$ is the data matrix of the hidden layer, as can be seen in

$$
\begin{aligned}
& X_{p}=\left(x_{p 1}, x_{p 2}, \ldots, x_{p_{n}}\right), \\
& H_{p}=\left(h_{p 1}, h_{p 2}, \ldots, h_{p l}\right), \\
& Y_{p}=\left(y_{p 1}, y_{p 2}, \ldots, y_{p m}\right),
\end{aligned}
$$

where $n, m, l$, and $p$ are the numbers of inputs, outputs, hidden layers, and the training samples, respectively.

(2) The outputs of the hidden layer are obtained by

$$
h_{p i}=f\left(\sum_{i=1}^{n} \omega_{i j} x_{p i}-a_{j}\right), \quad j=1,2, \ldots, l,
$$

where $\omega_{i j}$ is the connection between the input layer and the hidden layer.

(3) The predicted outputs of the BPNN can be obtained by

$$
\widehat{y}_{p k}=\sum_{j=1}^{l} h_{p i} \widehat{\omega}_{j k}-b_{k}, \quad k=1,2, \ldots, m,
$$

where $\widehat{\omega}_{j k}$ is the connection between the hidden layer and the output layer.

(4) According to the network forecast output $\hat{y}_{k}$ and the expected output $y_{k}$, calculate the network prediction error $e_{k}$.

$$
e_{k}=y_{p k}-\widehat{y}_{p k} .
$$

(5) The weights are updated according to the network prediction error $\left(e_{k}\right)$.

$$
\begin{aligned}
\omega_{i j} & =\omega_{i j}+\eta h_{p i}\left(1-h_{p i}\right) x(i) \sum_{k=1}^{m} \widehat{\omega}_{j k} e_{k}, \\
\widehat{\omega}_{j k} & =\widehat{\omega}_{j k}+\eta h_{p j} e_{k},
\end{aligned}
$$

where $\eta$ is the learning efficiency of BPNN.

(6) The thresholds are updated according to the network prediction error $\left(e_{k}\right)$.

$$
\begin{aligned}
& a_{j}=a_{j}+\eta h_{p j}\left(1-h_{p j}\right) \sum_{k=1}^{m} \widehat{\omega}_{j k} e_{k}, \\
& b_{k}=b_{k}+\eta e_{k} .
\end{aligned}
$$

(7) Return to step (2) if the optimization is still running.

It is noted that all the iterative processes of the BP neural network have a mean square error (MSE). In addition, the average accuracy of the prediction $(R)$ is defined by Kreith (2000). In other words, $R$ represents the goodness of fit, which is used to measure the correlation between prediction output data and training samples data. The closer it is to the value of 1 , the better the training network is.

$$
\begin{aligned}
\text { MSE } & =\frac{1}{m p} \sum_{p=1}^{p} \sum_{j=1}^{m}\left(\widehat{y}_{p j}-y_{p j}\right)^{2}, \\
R & =\frac{1}{P} \sum_{i=1}^{P} R_{i}=\frac{1}{P} \sum_{i=1}^{P} \frac{y_{p j}}{\widehat{y}_{p j}} .
\end{aligned}
$$

4.2. Genetic Algorithm. In this paper, the GA tool in MAT$\mathrm{LAB}$ is used for the optimization, which runs in the following principle:

(1) Initialize the population, calculate the fitness value, and find the best chromosome from the population.

(2) Iterative optimization:

(a) Select: first, the solution to the problem is encoded by using the floating-point encoding. This function selects the chromosomes in each generation population for subsequent crossover and mutation. The method used is the roulette selection method.

(b) Crossover: this function is a random selection of two chromosomes, according to determine the crossover probability to determine whether the cross, and the cross position is also random.

(c) Mutation: this function performs the mutation operation. The mutation chromosomes and mutated positions are randomly selected. Finally, it will check the feasibility of chromosomes; otherwise, it will be recompiled.

(d) Result analysis.

The optimal solution can be found after several generations. The establishment of BPNN approximate model and the numerical optimization of the genetic algorithm are shown in Figure 9. 


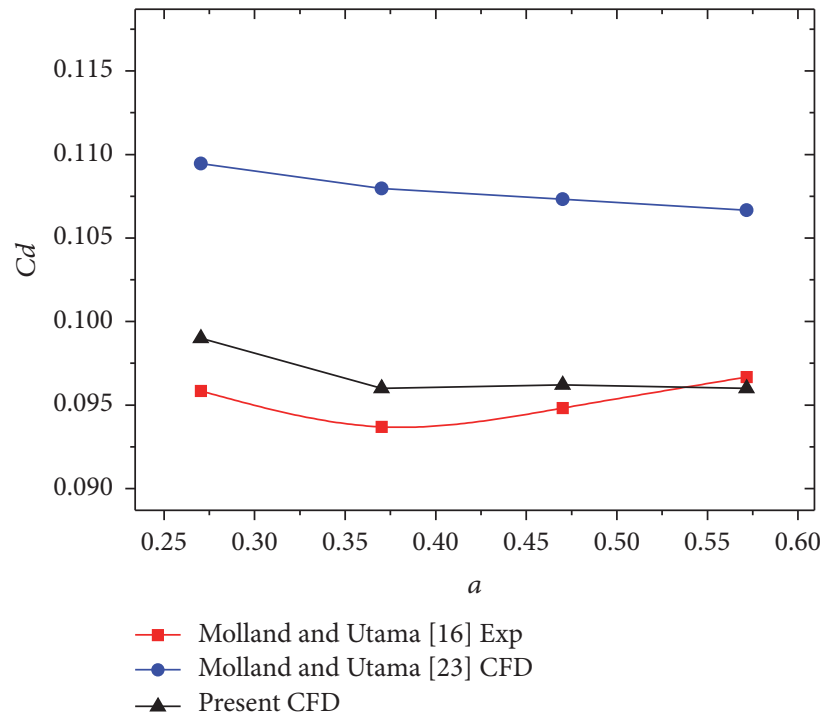

(a)

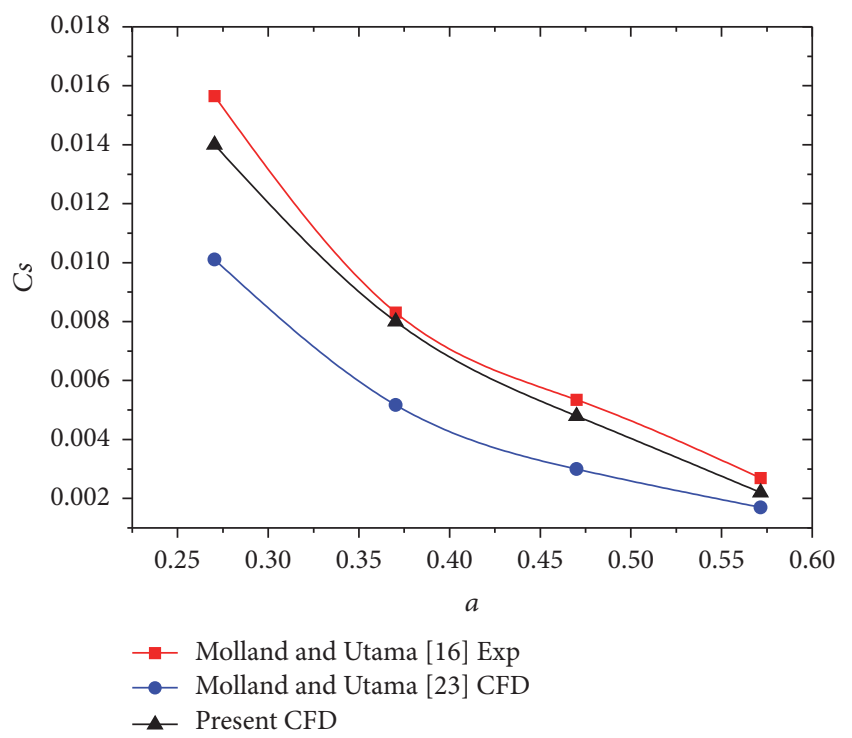

(b)

Figure 7: Results of the validation study: (a) coefficients of drag and (b) coefficients of side force.

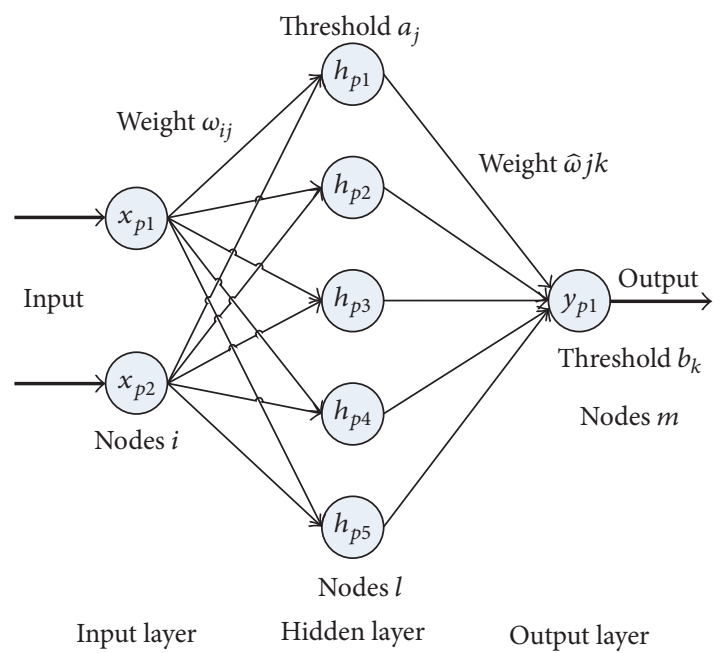

Figure 8: The topology of the BPNN.

\section{CFD Results}

5.1. Force Performance. In order to more intuitively show the influences of the fleet layout on the drags of the two AUVs, the coefficients of drags are expressed in a nondimensional drag ratio by dividing $C_{d 0}$ of a single $\mathrm{AUV}(0.0774$ in Table 2$)$ :

$$
\begin{gathered}
r_{l}=\frac{C_{d l}}{C_{d 0}}, \\
r_{f}=\frac{C_{d f}}{C_{d 0}}, \\
r_{\text {sum }}=\frac{C_{d l}+C_{d f}}{C_{d 0}} .
\end{gathered}
$$

The drag ratios for the 53 different fleet layouts are shown in Figure 10. The configuration of the AUV fleet can be divided into four regions depending on the drag ratios: the Parallel Region, the Pull Region, the Push Region, and the Tandem Region.

5.2. Parallel Region. The Parallel Region locates at $b=0$. AUVs in this region generate a higher drag than that in an infinite domain. Besides, the drag ratios for both AUVs are almost the same as each other due to the symmetrical geometric positions. For example, the drag ratios of AUVs at $a=0.20$ and $b=0.00$ are $5.52 \%$ higher than that of a single AUV. It can also be observed that as the latitudinal offset increases, the drag ratios gradually reduce. At $a=0.60$ and $b=0.00$, the drags of the AUVs are only approximately $0.6 \%$ higher than that of a single AUV. These results are consistent with previous studies $[16,26]$ which suggested a minimum latitudinal offset of $0.5 \mathrm{~L}$ to preclude the interactions between two AUVs.

The contours of pressure and velocity around the two parallel AUVs are shown in Figure 11. Three different latitudinal offsets, $a=0.2,0.4$, and 0.6 , are presented and compared. It can be seen from the figure that the velocity of the fluid is obviously increased in the range between the two AUVs. According to Bernoulli's Equation, larger fluid velocity corresponds to lower pressure, which can be verified by the pressure distributions in Figure 11(b). For example, in the pressure diagram of $a=0.2$, the pressure on the nose of the AUV is significantly reduced, which eventually leads to an increase in the drag. As $a$ increases, the interactions between the AUVs get weaker and the drag of the AUV is gradually restored to normal levels.

5.3. Tandem Region. The Tandem Region is where the follower AUV locates just behind the leader AUV $(a=0)$. The 


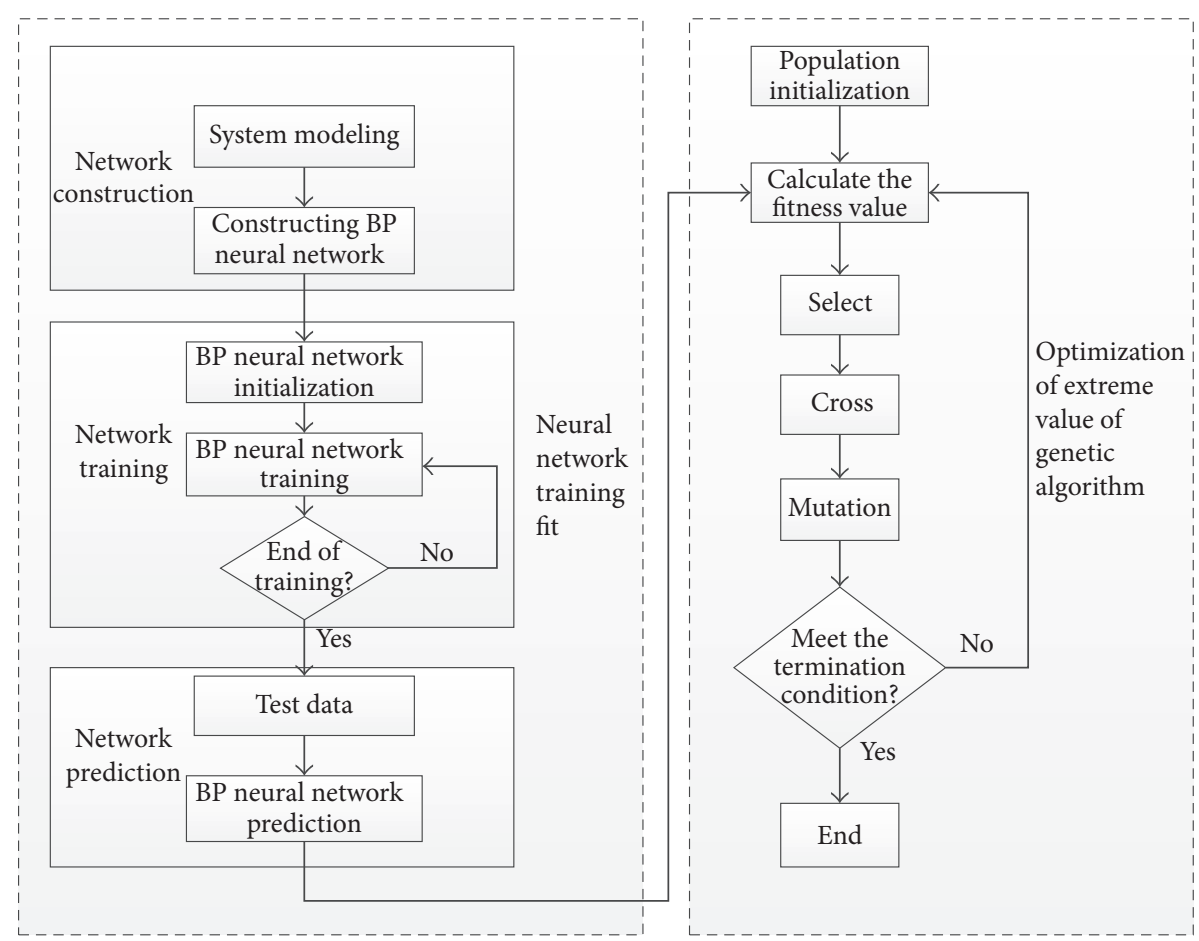

FIGURE 9: Neural network and genetic algorithm flowchart.

\begin{tabular}{|c|c|c|c|c|c|c|c|c|c|c|}
\hline & $b=0.00$ & $b=0.25$ & $b=0.50$ & $b=0.75$ & $b=1.00$ & $b=1.25$ & $b=1.50$ & $b=1.75$ & $b=2.00$ & \\
\hline$a=0.00$ & & & & & & $\begin{array}{l}0 . / 910 / \\
1.0972\end{array}$ & $\begin{array}{l}0.9392 / \\
0.9748\end{array}$ & $\begin{array}{c}0.9757 / \\
0.9602\end{array}$ & $\begin{array}{l}0.9885 \\
0.9629\end{array}$ & $\begin{array}{l}\text { Tandem } \\
\text { region }\end{array}$ \\
\hline$a=0.10$ & & & & & & $\begin{array}{c}0.8245 / \\
1.1725\end{array}$ & $\begin{array}{l}0.9432 / \\
1.0604\end{array}$ & $\begin{array}{l}0.9765 / \\
1.0296\end{array}$ & $\begin{array}{c}0.9887 / \\
1.0188\end{array}$ & \\
\hline$a=0.20$ & & & & 0.5041 & 1.2507 & $\begin{array}{l}0.8671 / \\
1.1107\end{array}$ & $\begin{array}{c}0.9450 / \\
1.0398\end{array}$ & $\begin{array}{l}0.9728 / \\
1.0150\end{array}$ & $\begin{array}{c}0.9841 / \\
1.0050\end{array}$ & \\
\hline$a=0.30$ & & & & & 1.0920 & $0.9135 /$ & 1.0297 & $\begin{array}{l}0.9761 / \\
1.0118\end{array}$ & $\begin{array}{l}0.9852 / \\
1.0037\end{array}$ & \\
\hline$a=0.40$ & & & & & 1.0283 & $\begin{array}{r}0.9487 / \\
1.0354\end{array}$ & $\begin{array}{l}0.9669 / \\
1.0200\end{array}$ & $\begin{array}{l}0.9800 / \\
1.0086\end{array}$ & $\begin{array}{l}0.9871 / \\
1.0025\end{array}$ & \\
\hline$a=0.50$ & 1.0095 & & 0.8506 & 0.9000 & $\begin{array}{l}1.0243 / \\
1.0032\end{array}$ & $\begin{array}{c}0.9723 / \\
1.0146\end{array}$ & $\begin{array}{l}0.9769 \\
1.0118\end{array}$ & $\begin{array}{c}0.9843 / \\
1.0057\end{array}$ & $\begin{array}{l}0.9891 / \\
1.0016\end{array}$ & \\
\hline$a=0.60$ & & & 126 & & $\begin{array}{l}1.0724 \\
0.924\end{array}$ & $\begin{array}{l}0.9873 / \\
1.0018\end{array}$ & $\begin{array}{l}0.9850 / \\
1.0052\end{array}$ & $\begin{array}{l}0.9883 / \\
1.0029\end{array}$ & $\begin{array}{l}0.9911 / \\
1.0000\end{array}$ & \\
\hline & $\begin{array}{l}\text { Parallel } \\
\text { region }\end{array}$ & \multicolumn{4}{|c|}{ Pull region } & \multicolumn{4}{|c|}{ Push region } & \\
\hline
\end{tabular}

FigURE 10: Drag ratios for different AUV fleet layouts.

drag ratios of the AUVs in this region are greatly reduced, especially for the leader AUV. For example, at $a=0.00$ and $b=1.50$, the drag reduction is $6.18 \%$ for the leader AUV and is $2.52 \%$ for the follower AUV. It should be noted that the follower AUV experiences an $9.72 \%$ increase in drag at $a=0.00$ and $b=1.25$, but the total drag of the AUV fleet reduces.

The contours of pressure and velocity around the two tandem AUVs are shown in Figure 12. Three different longitudinal offsets, $b=1.25,1.50$, and 1.75 , are presented and compared. When the follower AUV is located in tandem in the wake of the leader AUV, a certain amount of pressure is recovered on the tail of the leader AUV, which contributes to the reduction of the pressure drag of the leader AUV, while for the follower AUV, the velocity of the upstream flow is reduced by the leader AUV, resulting in a reduction of the nose pressure and a decrease in final resistance.

5.4. Pull Region. The Pull Region is where the drag of the leader AUV is increased and that of the follower AUV is 

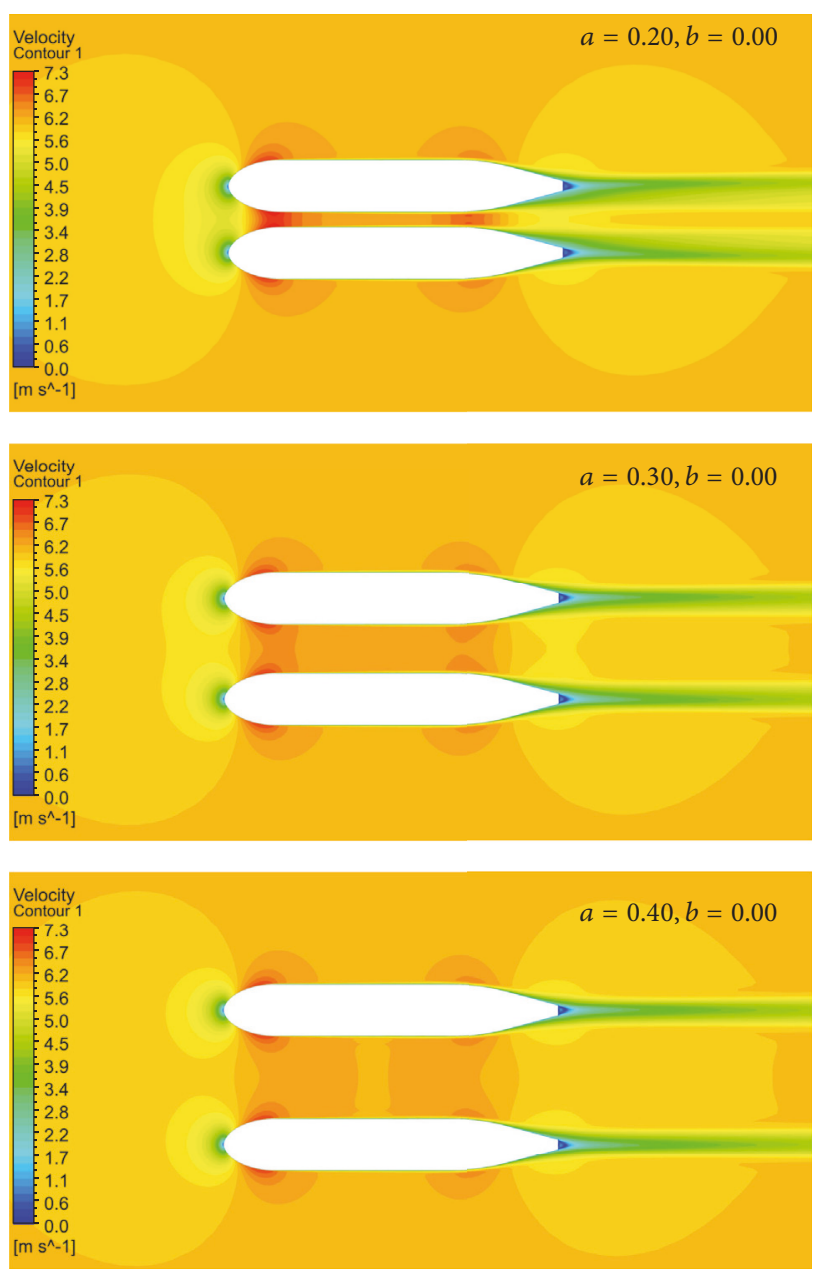

(a)
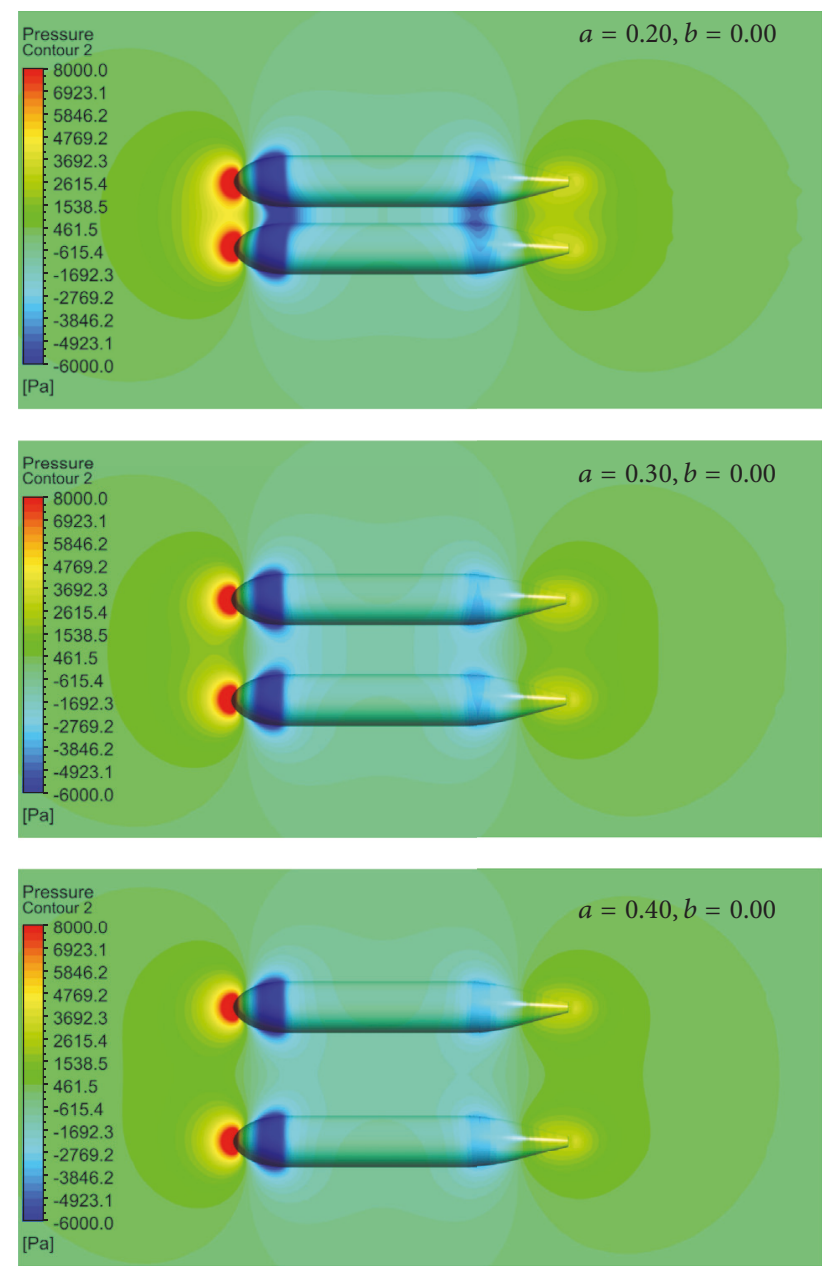

(b)

FIGURE 11: Contours of (a) velocity and (b) pressure around two parallel AUVs at different latitudinal offsets.

reduced. The Pull Region mainly locates between $0.20 \leq a \leq$ 0.60 and $0.25 \leq b \leq 1.00$. In this region, it is likely that the leader AUV pulls the follower AUV to move, so that the drag of the leader AUV is increased and the drag of the follower AUV changes oppositely.

The contours of pressure and velocity around the two AUVs in the Pull Region are shown in Figure 13. Three different locations with $a=0.2,0.3$, and $0.4, b=0.25$, 0.50 , and 0.75 , respectively, are presented and compared. The longitudinal offset is within one AUV length and the nose of the follower AUV locates at the lower pressure region by the side of the leader AUV. Under the effects of the follower AUV, the pressure on the side surface of the leader AUV is reduced, resulting in an augment in the drag. Besides, as the $a$ increases, the interactions between the two AUVs are weaker and the variation in the drags of both AUVs is smaller.

5.5. Push Region. The Push Region is where the drag of the leader AUV is reduced and that of the follower AUV is augmented. The Push Region mainly locates between $0.10 \leq$ $a \leq 0.60$ and $b \geq 1.25$. In this region, it is likely that the follower AUV pushes the leader AUV to move, so that the drag of the leader AUV is reduced and the drag of the follower AUV changes oppositely.

The contours of pressure and velocity around the two AUVs in the Push Region are shown in Figure 14. Three different locations with $a=0.2,0.3$, and $0.4, b=1.25$, 1.50 , and 1.75 , respectively, are presented and compared. The reason for the variation of the drags on the two AUVs can be explained. The obstacle flow around the nose of the follower AUV hinders the separation on the tail of the leader AUV; therefore, a certain amount of pressure is recovered on the tail of the leader AUV, which contributes to a reduction of the pressure drag of the leader AUV. A reaction force is then applied to the follower AUV, leading to an increase in the drag on the follower AUV.

5.6. Data for the BPNN Training Samples. The predicted drag ratios for the AUV fleet obtained by CFD are given in Table 3 . It should be noted that 10 samples for $a \leq 0.20$ and $b \leq 1.00$ are added to complete an orthographical sample domain. Since it is nonphysical to arrange the two AUVs in this area, a 

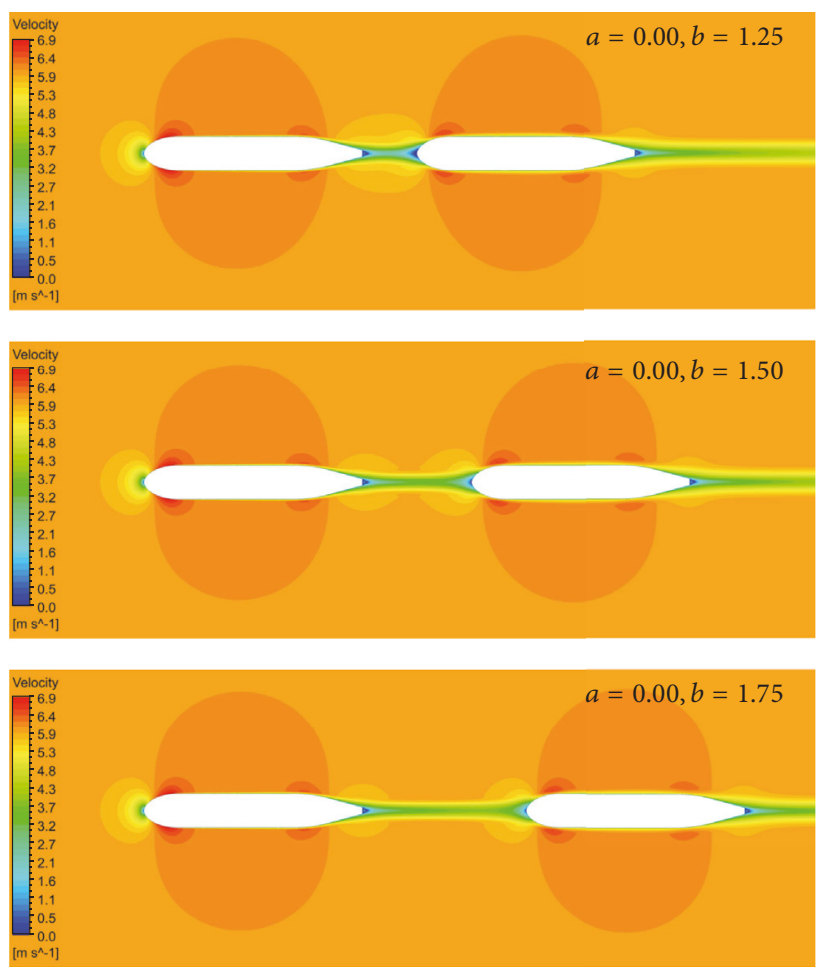

(a)
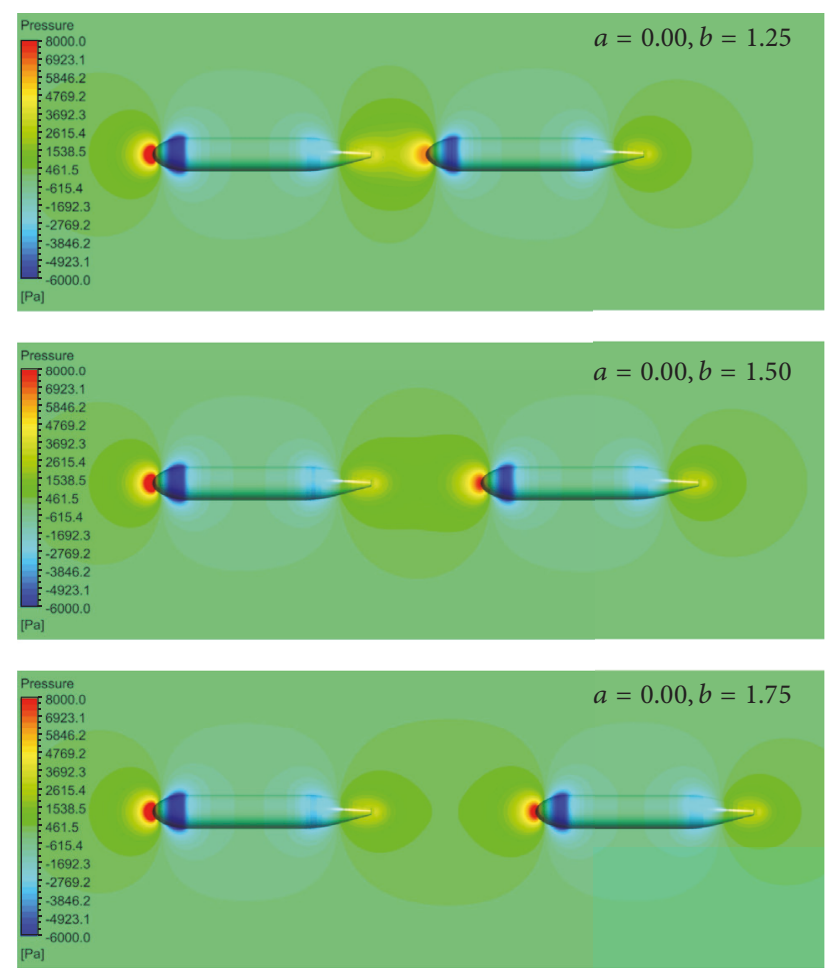

(b)

FIGURE 12: Contours of (a) velocity and (b) pressure around two parallel AUVs at different latitudinal offsets.

high drag ratio of 2.0000 is assigned to the AUV which locates in this region. Therefore, there are 63 samples in total for the training of the BPNN.

\section{Neural Network and Genetic Algorithm Optimization}

6.1. Optimization of the AUV Fleet. When the AUV fleet executes tasks underwater, a minimum drag is expected so that the fleet will work for a longer time and a wider range. Therefore, the layout of the AUV fleet should be optimized to obtain a minimum drag.

6.1.1. Neural Network Training. Using the data in Table 3, training of the BPNN is carried out until the network meets the intended target. A comparison between the predicted data and the expected data is shown in Figure 15. The training network predicts drag ratios with good agreement with the CFD data. Therefore, it can be said that the BPNN successfully model and predict the drag ratio of the AUV fleet.

\subsubsection{Comparisons of the Optimal Drag between CFD and} $B P N N$. A CFD simulation of the AUV fleet for the optimal parameters is carried out for the comparisons between the two methods. The optimal results are shown in Table 4. The results of the BPNN and the CFD method are very close, with a relative error of $0.66 \%$, which further validates the accuracy of the BPNN. The optimal drag ratio is obtained at $a=0.00$ and $b=1.21$, where the follower AUV is directly behind the Leading AUV, and the optimal drag ratio of the AUV fleet is 1.8825 , which means that the total drag of the AUV fleet is reduced by approximately $12 \%$.

To further investigate the flow structures of the optimal fleet layout, Figure 16 compares the pressure contours around the optimal AUV fleet and that of a single AUV. The most obvious difference between these two cases is the pressure distribution on the tail of the leader AUV. Due to the block effect of the follower AUV, the pressure on the tail of the leader AUV is recovered. This variation can be clearly observed in the pressure distributions in Figure 17.

6.2. Optimization of the Follower AUV. Although the optimal fleet layout analyzed in Section 6.2 characterizes with the minimum drag, the drag of the follower AUV is not the optimal value. Among the AUVs in a fleet, it is possible that leader AUV is designed with high propulsive performance and the other follower AUVs obtain lower propulsion costs at the expense of the leader AUV. Therefore, the optimal layout of the fleet for the best performance of the follower AUV should be studied.

6.2.1. Neural Network Training. Using the data in Table 3, training of the BPNN is carried out until the network meets the intended target. A comparison between the predicted data and the expected data for the follower AUV is shown in Figure 18. As can be seen from the figure, the training network predicts drag ratios with good agreement with the CFD data. 
TABLE 3: CFD data for the training samples.

\begin{tabular}{|c|c|c|c|c|c|}
\hline Sample & $a$ & $b$ & $r_{l}$ & $r_{f}$ & $r_{\text {sum }}$ \\
\hline (1) & 0.00 & 0.00 & 2.0000 & 2.0000 & 4.0000 \\
\hline (2) & 0.00 & 0.25 & 2.0000 & 2.0000 & 4.0000 \\
\hline (3) & 0.00 & 0.50 & 2.0000 & 2.0000 & 4.0000 \\
\hline (4) & 0.00 & 0.75 & 2.0000 & 2.0000 & 4.0000 \\
\hline (5) & 0.00 & 1.00 & 2.0000 & 2.0000 & 4.0000 \\
\hline (6) & 0.00 & 1.25 & 0.7910 & 1.0972 & 1.8882 \\
\hline (7) & 0.00 & 1.50 & 0.9392 & 0.9748 & 1.9141 \\
\hline (8) & 0.00 & 1.75 & 0.9757 & 0.9602 & 1.9359 \\
\hline (9) & 0.00 & 2.00 & 0.9885 & 0.9629 & 1.9514 \\
\hline (10) & 0.10 & 0.00 & 2.0000 & 2.0000 & 4.0000 \\
\hline (11) & 0.10 & 0.25 & 2.0000 & 2.0000 & 4.0000 \\
\hline (12) & 0.10 & 0.50 & 2.0000 & 2.0000 & 4.0000 \\
\hline (13) & 0.10 & 0.75 & 2.0000 & 2.0000 & 4.0000 \\
\hline (14) & 0.10 & 1.00 & 2.0000 & 2.0000 & 4.0000 \\
\hline (15) & 0.10 & 1.25 & 0.8245 & 1.1725 & 1.9970 \\
\hline (16) & 0.10 & 1.50 & 0.9432 & 1.0604 & 2.0036 \\
\hline (17) & 0.10 & 1.75 & 0.9765 & 1.0296 & 2.0061 \\
\hline (18) & 0.10 & 2.00 & 0.9887 & 1.0188 & 2.0075 \\
\hline (19) & 0.20 & 0.00 & 1.0552 & 1.0552 & 2.1104 \\
\hline (20) & 0.20 & 0.25 & 1.7136 & 0.3598 & 2.0733 \\
\hline (21) & 0.20 & 0.50 & 1.5153 & 0.5364 & 2.0517 \\
\hline (22) & 0.20 & 0.75 & 1.4027 & 0.5841 & 1.9868 \\
\hline (23) & 0.20 & 1.00 & 0.7591 & 1.2507 & 2.0098 \\
\hline (24) & 0.20 & 1.25 & 0.8671 & 1.1107 & 1.9778 \\
\hline (25) & 0.20 & 1.50 & 0.9450 & 1.0398 & 1.9848 \\
\hline (26) & 0.20 & 1.75 & 0.9728 & 1.0150 & 1.9879 \\
\hline (27) & 0.20 & 2.00 & 0.9841 & 1.0050 & 1.9891 \\
\hline (28) & 0.30 & 0.00 & 1.0247 & 1.0247 & 2.0494 \\
\hline (29) & 0.30 & 0.25 & 1.4041 & 0.6326 & 2.0367 \\
\hline (30) & 0.30 & 0.50 & 1.3595 & 0.6702 & 2.0297 \\
\hline (31) & 0.30 & 0.75 & 1.1989 & 0.7884 & 1.9873 \\
\hline (32) & 0.30 & 1.00 & 0.9257 & 1.0920 & 2.0177 \\
\hline (33) & 0.30 & 1.25 & 0.9135 & 1.0671 & 1.9806 \\
\hline (34) & 0.30 & 1.50 & 0.9557 & 1.0297 & 1.9853 \\
\hline (35) & 0.30 & 1.75 & 0.9761 & 1.0118 & 1.9879 \\
\hline (36) & 0.30 & 2.00 & 0.9852 & 1.0037 & 1.9889 \\
\hline (37) & 0.40 & 0.00 & 1.0146 & 1.0146 & 2.0292 \\
\hline (38) & 0.40 & 0.25 & 1.2386 & 0.7859 & 2.0245 \\
\hline (39) & 0.40 & 0.50 & 1.2489 & 0.7770 & 2.0259 \\
\hline (40) & 0.40 & 0.75 & 1.1302 & 0.8617 & 1.9920 \\
\hline (41) & 0.40 & 1.00 & 0.9947 & 1.0283 & 2.0229 \\
\hline (42) & 0.40 & 1.25 & 0.9487 & 1.0354 & 1.9841 \\
\hline (43) & 0.40 & 1.50 & 0.9669 & 1.0200 & 1.9869 \\
\hline (44) & 0.40 & 1.75 & 0.9800 & 1.0086 & 1.9886 \\
\hline$(45)$ & 0.40 & 2.00 & 0.9871 & 1.0025 & 1.9896 \\
\hline (46) & 0.50 & 0.00 & 1.0094 & 1.0095 & 2.0189 \\
\hline (47) & 0.50 & 0.25 & 1.1488 & 0.8699 & 2.0187 \\
\hline (48) & 0.50 & 0.50 & 1.1753 & 0.8506 & 2.0259 \\
\hline
\end{tabular}


TABle 3: Continued.

\begin{tabular}{|c|c|c|c|c|c|}
\hline Sample & $a$ & $b$ & $r_{l}$ & $r_{f}$ & $r_{\text {sum }}$ \\
\hline (49) & 0.50 & 0.75 & 1.0950 & 0.9000 & 1.9951 \\
\hline (50) & 0.50 & 1.00 & 1.0243 & 1.0032 & 2.0275 \\
\hline (51) & 0.50 & 1.25 & 0.9723 & 1.0146 & 1.9868 \\
\hline (52) & 0.50 & 1.50 & 0.9769 & 1.0118 & 1.9887 \\
\hline (53) & 0.50 & 1.75 & 0.9843 & 1.0057 & 1.9900 \\
\hline (54) & 0.50 & 2.00 & 0.9891 & 1.0016 & 1.9907 \\
\hline (55) & 0.60 & 0.00 & 1.0062 & 1.0065 & 2.0127 \\
\hline (56) & 0.60 & 0.25 & 1.0963 & 0.9170 & 2.0132 \\
\hline (57) & 0.60 & 0.50 & 1.1263 & 0.8991 & 2.0253 \\
\hline (58) & 0.60 & 0.75 & 1.0724 & 0.9245 & 1.9969 \\
\hline (59) & 0.60 & 1.00 & 1.0327 & 0.9907 & 2.0235 \\
\hline (60) & 0.60 & 1.25 & 0.9873 & 1.0018 & 1.9891 \\
\hline (61) & 0.60 & 1.50 & 0.9850 & 1.0052 & 1.9903 \\
\hline (62) & 0.60 & 1.75 & 0.9883 & 1.0029 & 1.9912 \\
\hline (63) & 0.60 & 2.00 & 0.9911 & 1.0000 & 1.9911 \\
\hline
\end{tabular}
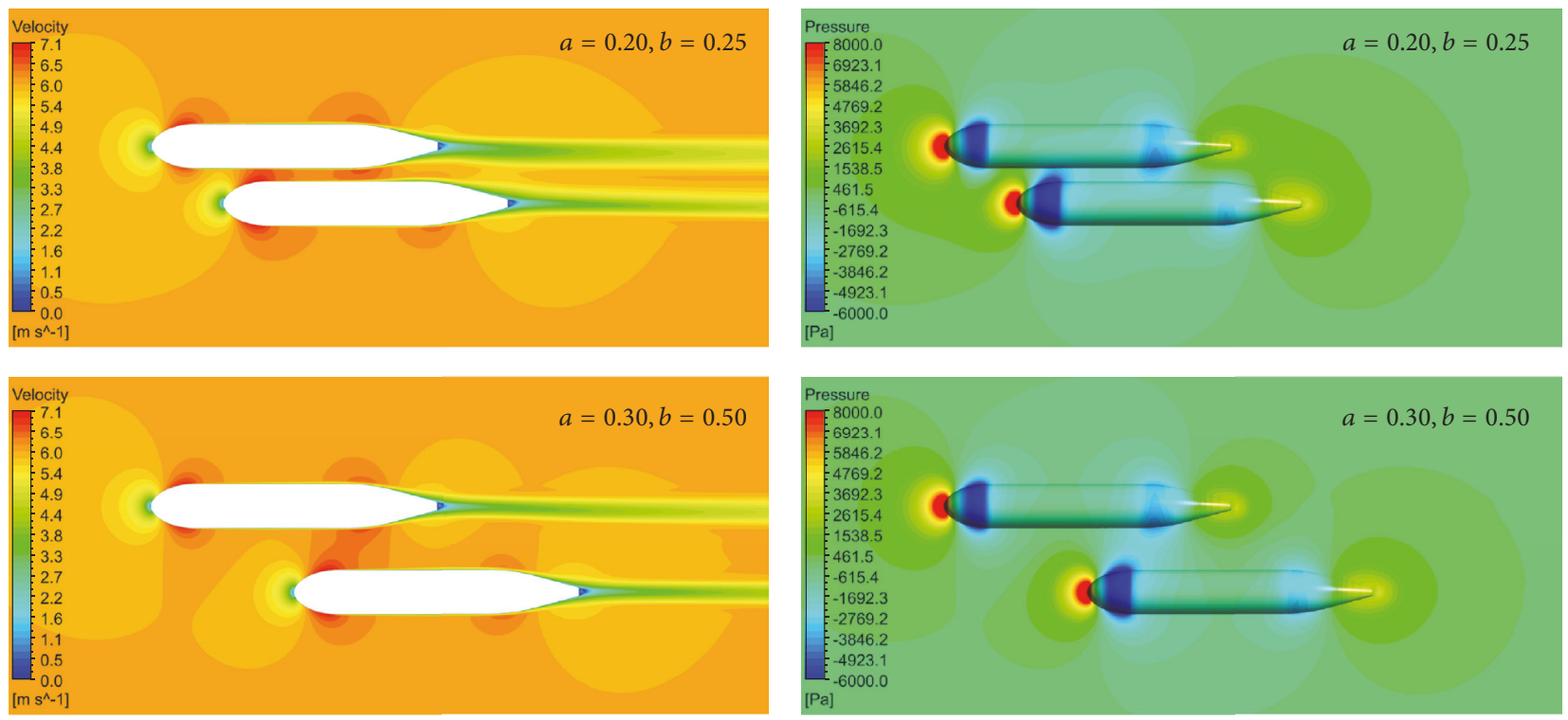

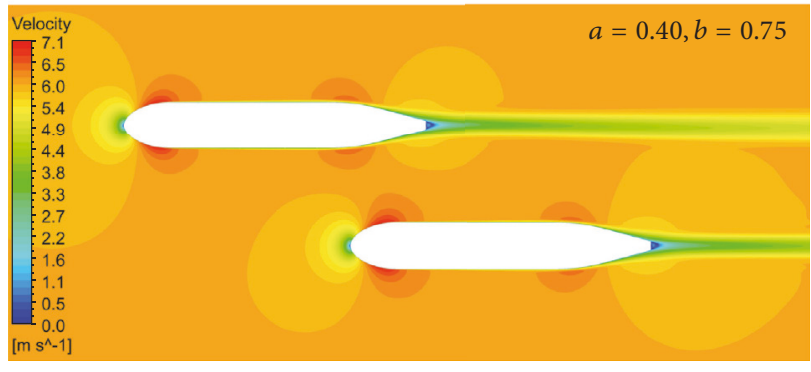

(a)

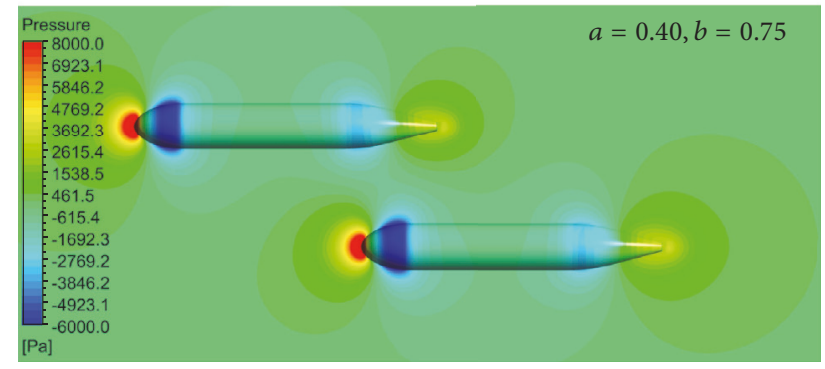

(b)

FIGURE 13: Contours of (a) velocity and (b) pressure around two AUVs in the Pull Region. 

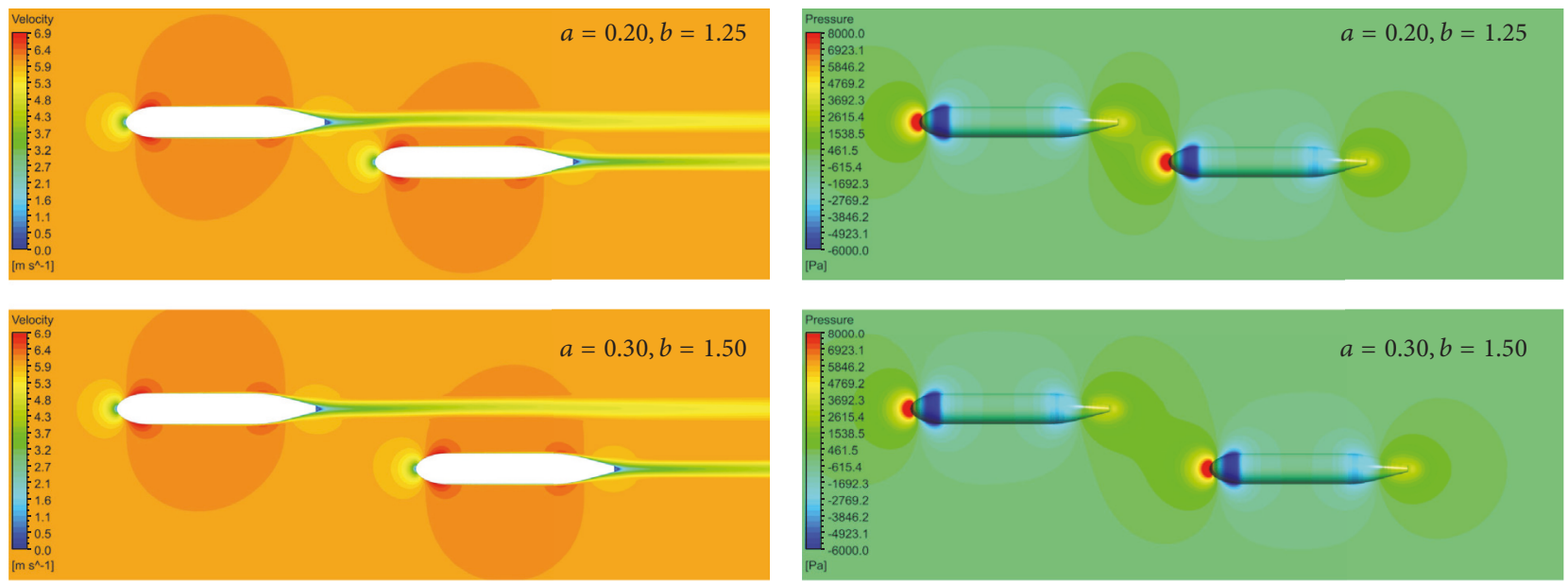

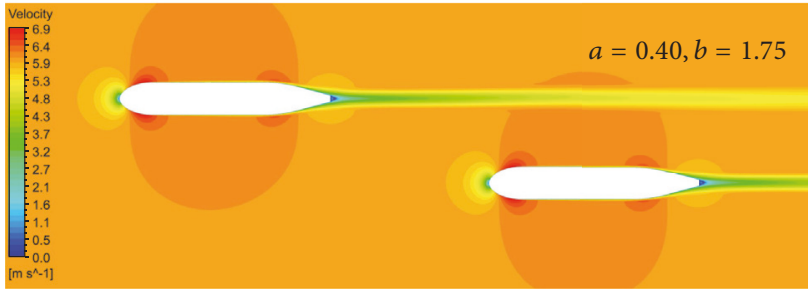

(a)

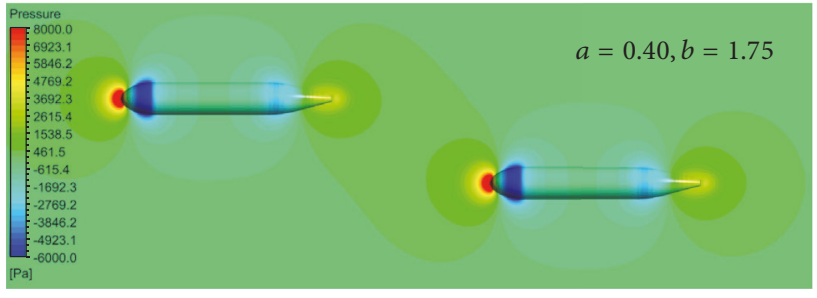

(b)

FIGURE 14: Contours of (a) velocity and (b) pressure around two AUVs in the Pull Region.

TABLE 4: Comparisons of the optimal drag ratio of the AUV fleet between CFD and BPNN.

\begin{tabular}{lcccc}
\hline$a$ & $b$ & BPNN & CFD & Relative error (\%) \\
\hline 0.00 & 1.21 & 1.8949 & 1.8825 & $0.66 \%$ \\
\hline
\end{tabular}

TABLE 5: Comparisons of the optimal drag ratio of the follower AUV between CFD and BPNN.

\begin{tabular}{lcccc}
\hline$a$ & $b$ & BPNN & CFD & Relative error (\%) \\
\hline 0.22 & 0.29 & 0.3481 & 0.3417 & $1.87 \%$ \\
\hline
\end{tabular}

6.2.2. Comparisons of the Optimal Drag between CFD and $B P N N$. Based on the network after the training process, the optimal output drag ratio of the follower AUV is predicted using the BPNN model. Typically, a CFD simulation for the optimal parameters is carried out for the comparisons between the two methods. The optimal results are shown in Table 5. Table 5 suggests that the results of the BPNN and the CFD method are very close, with a relative error of $1.87 \%$, which further validates the accuracy of the BPNN. The optimal drag ratio is obtained at $a=0.00$ and $b=1.21$, where the AUV fleet is in the Pull Region. The optimal drag ratio of the follower AUV is 0.3417, which means that the drag of the follower AUV is reduced by approximately $66 \%$.

Figure 19 compares the pressure contours around the optimal AUV fleet and that of a single AUV. The most obvious difference between these two cases is the pressure distribution around the nose of the follower AUV. At this position, the

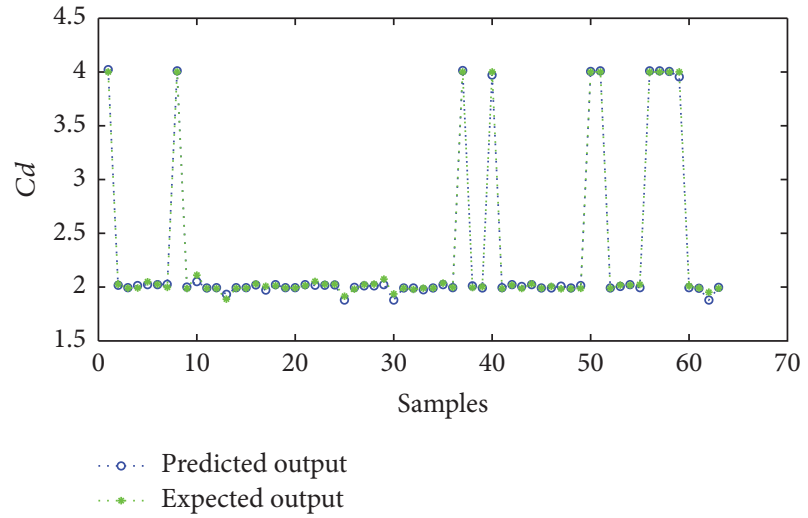

FIGURE 15: Comparisons of the drag ratio of the AUV fleet by BPNN and CFD.

nose of the follower AUV is influenced by the negative pressure on the inside surface of the leader AUV, so that a smaller pressure is obtained. This can also be observed in the pressure distributions in Figure 20.

\section{Conclusions}

The drag of the AUV fleet significantly influences the operating time and range of underwater vehicles. In this study, an optimization method is proposed to find the optimal layout of the AUV fleet which has the minimum drag. A combined method of CFD simulation, BPNN, and GA method is 


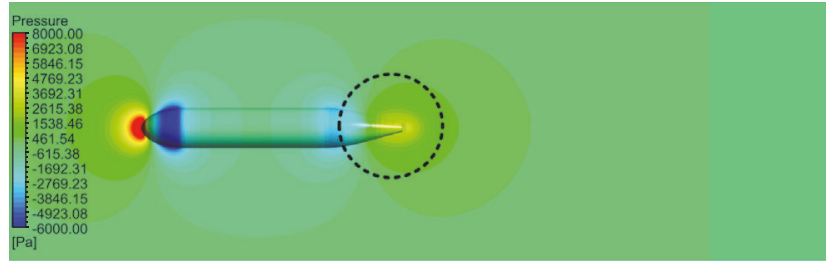

(a)

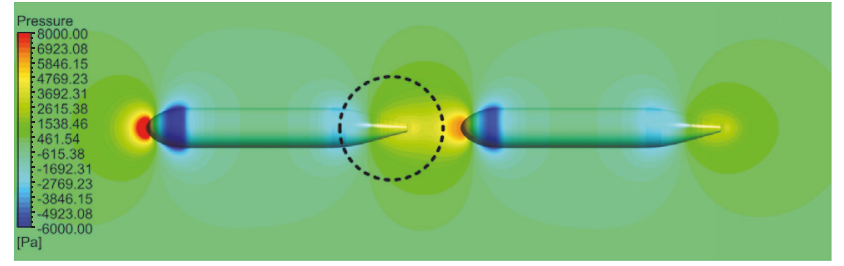

(b)

FIGURE 16: Contours of pressure around the AUV: (a) single AUV and (b) optimal AUV fleet.

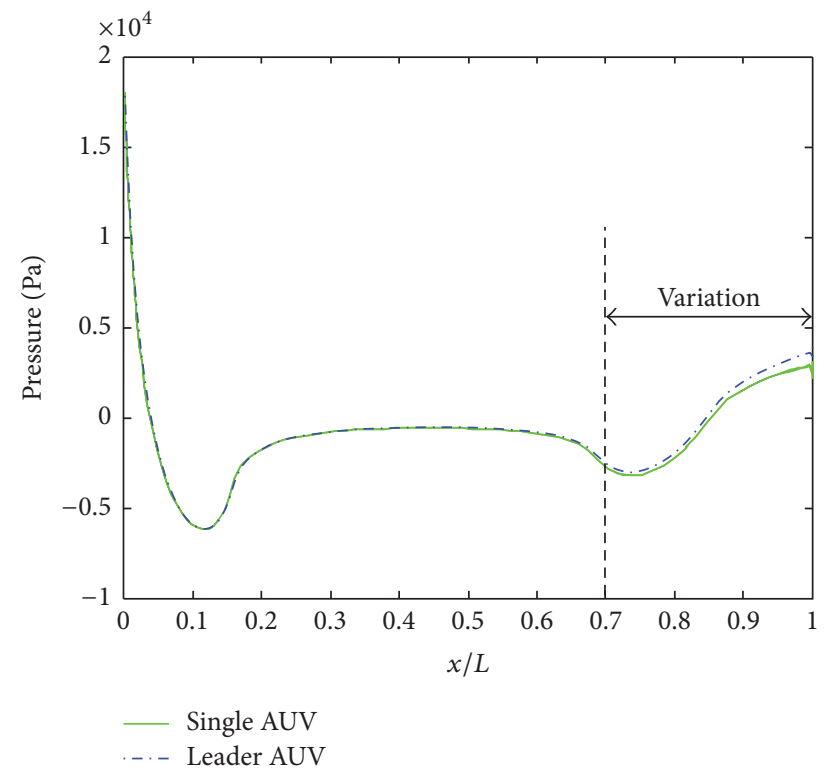

FIGURE 17: Pressure distributions along the longitudinal direction of the AUV: (a) single AUV and (b) optimal AUV fleet.

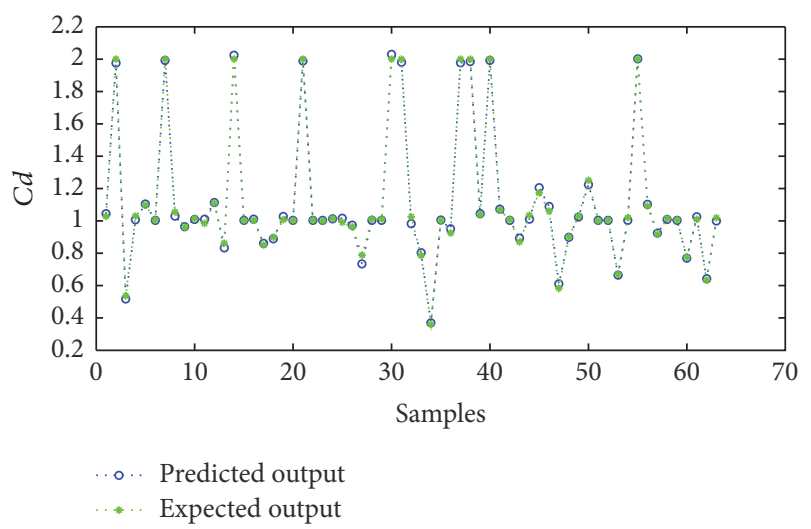

FIGURE 18: Comparisons of the drag ratio of the follower AUV by BPNN and CFD.

utilized for the optimization of the AUV fleet. Important conclusions of this study include the following:

(1) The CFD results show that the drags of the AUVs in the fleet are determined by the relative position of the two AUVs. The layout of the fleet can be categorized into four

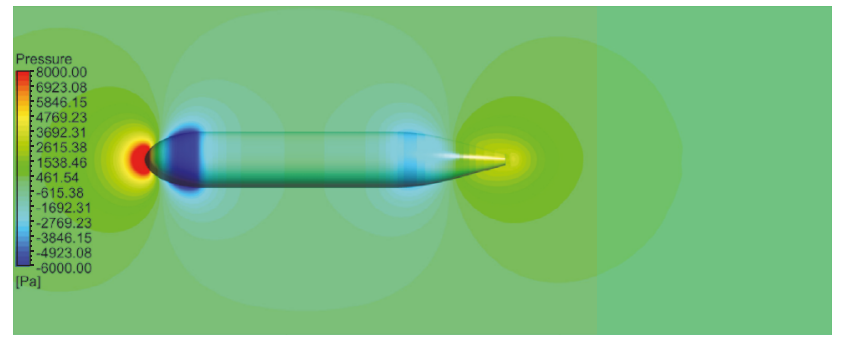

(a)

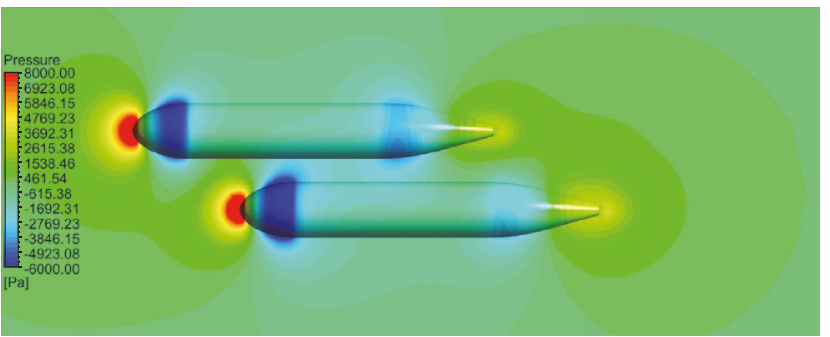

(b)

FIGURE 19: Contours of pressure around the AUV: (a) single AUV and (b) optimal AUV fleet.

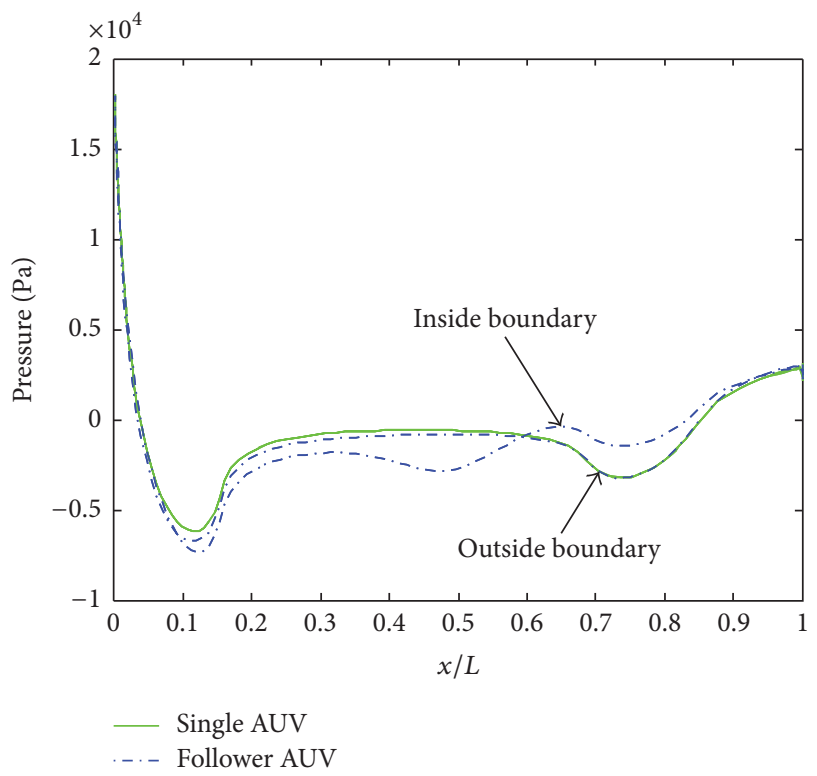

FIgURE 20: Pressure distributions along the longitudinal direction of the AUV: (a) single AUV and (b) optimal AUV fleet. 
different regions based on the drag behavior of the two AUVs: the Parallel Region, the Tandem Region, the Pull Region, and the Push Region. The variation of the drags of the AUVs is the result of the interacting flow and pressure change on the two AUVs.

(2) The optimal layout parameters for the minimum fleet drag are $a=0.00$ and $b=1.21$, where the fleet is in the Tandem Region and predicts a drag approximately $12 \%$ lower than that of a single AUV.

(3) The optimal layout parameters for the minimum drag of the follower AUV are $a=0.22$ and $b=0.29$, where the fleet is in the Pull Region and predicts a drag approximately $66 \%$ lower than that of a single AUV.

\section{Nomenclature}

AUV: Autonomous underwater vehicles

BPNN: Back-propagation neural network

CFD: Computational fluid dynamics

GA: Genetic algorithm

D: $\quad$ Maximum diameter of the AUV

L: $\quad$ Length of the AUV

S: $\quad$ Maximum cross-sectional area of the $\left(\mathrm{m}^{2}\right)$

$U: \quad$ Velocity of the AUV

$\rho$ : $\quad$ Density of seawater

$y^{+}: \quad y$-plus value

$a, b$ : Normalized position of the follower AUV

$x_{f}, y_{f}$ : Position of the follower AUV

$C_{d l}: \quad$ Drag coefficient of the leader AUV

$C_{d f}$ : Drag coefficient of the follower AUV

$C_{d \text { sum }}$ : Drag coefficient of the fleet

$r_{l}: \quad$ Drag ratio of the leader AUV

$r_{f}$ : Drag ratio of the follower AUV

$r_{\text {sum }}:$ Drag ratio of the fleet

$k$ : $\quad$ Turbulence kinetic energy

$\omega: \quad$ Specific rate of dissipation.

\section{Conflicts of Interest}

The authors declare that there are no conflicts of interest regarding the publication of this paper.

\section{Acknowledgments}

This research was supported by the National Science Foundation of China (Grants nos. 51179159 and 61572404).

\section{References}

[1] X. Li, H. Zhu, G. Chen, and R. Zhang, "Optimal maintenance strategy for corroded subsea pipelines," Journal of Loss Prevention in the Process Industries, vol. 49, pp. 145-154, 2017.

[2] X. Wang, C. Yang, Z. Ju, H. Ma, and M. Fu, "Robot manipulator self-identification for surrounding obstacle detection," Multimedia Tools Applications, pp. 1-26, 2016.

[3] D. Edwards, T. Bean, D. Odell, and M. Anderson, "A leaderfollower algorithm for multiple AUV formations," in Proceedings of the IEEE/OES Autonomous Underwater Vehicles, pp. 4046, Sebasco, Me, USA, June 2004.
[4] S. Botelho, R. Neves, and B. L. Taddei, "Localization of a fleet of AUVs using visual maps," in Proceedings of the Europe Oceans 2005, Brest, France, June 2005.

[5] R. M. Alexander, "Hitching a lift hydrodynamically-In swimming, flying and cycling," Journal of Biology, vol. 3, no. 2, article 7,2004.

[6] P. Jagadeesh, K. Murali, and V. G. Idichandy, "Experimental investigation of hydrodynamic force coefficients over AUV hull form," Ocean Engineering, vol. 36, no. 1, pp. 113-118, 2009.

[7] P. Stevenson, M. Furlong, and D. Dormer, "AUV shapes Combining the practical and hydrodynamic considerations," in Proceedings of the Europe OCEANS 2007, pp. 1-6, June 2007.

[8] A. Alvarez, V. Bertram, and L. Gualdesi, "Hull hydrodynamic optimization of autonomous underwater vehicles operating at snorkeling depth," Ocean Engineering, vol. 36, no. 1, pp. 105-112, 2009.

[9] H. Kim, C. Yang, R. Löhner, and F. Noblesse, "A practical hydrodynamic optimization tool for the design of a monohull ship," in Proceedings of the 18th International Offshore and Polar Engineering Conference, ISOPE 2008, pp. 98-107, July 2008.

[10] T.-H. Joung, K. Sammut, F. He, and S.-K. Lee, "Shape optimization of an autonomous underwater vehicle with a ducted propeller using computational fluid dynamics analysis," International Journal of Naval Architecture and Ocean Engineering, vol. 4, no. 1, pp. 44-56, 2012.

[11] C. Sun, B. Song, P. Wang, and X. Wang, "Shape optimization of blended-wing-body underwater glider by using gliding range as the optimization target," International Journal of Naval Architecture \& Ocean Engineering, vol. 9, no. 6, pp. 693-707, 2017.

[12] K. Elsayed and C. Lacor, "The Effect of The Cyclone Separator Cone Height on The Performance Using Artificial Neural Network Model and CFD Simulations," in Proceedings of the International Symposium on Experimental and Computational Aerothermodynamics of Internal Flows, Brussels, Belgium, 2011.

[13] C. Shen, L. Wang, and Q. Li, "Optimization of injection molding process parameters using combination of artificial neural network and genetic algorithm method," Journal of Materials Processing Technology, vol. 183, no. 2-3, pp. 412-418, 2007.

[14] H. Safikhani, A. Abbassi, A. Khalkhali, and M. Kalteh, "Multiobjective optimization of nanofluid flow in flat tubes using CFD, Artificial Neural Networks and genetic algorithms," Advanced Powder Technology, vol. 25, no. 5, pp. 1608-1617, 2014.

[15] H. Avc1, D. Kumlutaş, Ö. Özer, and M. Özşen, “Optimisation of the design parameters of a domestic refrigerator using CFD and artificial neural networks," International Journal of Refrigeration, vol. 67, pp. 227-238, 2016.

[16] A. F. Molland and I. K. A. P. Utama, Wind Tunnel Investigation of a Pair of Ellipsoids in Close Proximity, University of Southampton, 1997.

[17] D. F. Myring, "A theoretical study of body drag in subcritical axisymmetric flow," Aeronautical Quarterly, vol. 27, no. 3, pp. 186-194, 1976.

[18] I. Ansys, "Fluent 15.0 user's guide," 2014.

[19] W. Tian, Z. Mao, and Y. Li, "Numerical Simulations of a VAWT in the Wake of a Moving Car," Energies, vol. 10, no. 4, p. 478, 2017.

[20] W. Tian, Z. Mao, X. An, B. Zhang, and H. Wen, "Numerical study of energy recovery from the wakes of moving vehicles on highways by using a vertical axis wind turbine," Energy, vol. 141, pp. 715-728, 2017. 
[21] Y. Qiu, Z. Liu, X. Chen, and C. Zhan, "RETRACTED ARTICLE: Numerical calculation of maneuvering hydrodynamic forces of drift ship based on SST k-w turbulence model," in Proceedings of the International Conference on Computer Engineering and Technology, pp. V5285-V5288, 2010.

[22] W. Tian, Z. Mao, B. Zhang, and Y. Li, "Shape optimization of a Savonius wind rotor with different convex and concave sides," Journal of Renewable Energy, vol. 117, pp. 287-299, 2018.

[23] A. F. Molland and I. K. A. P. Utama, "Experimental and numerical investigations into the drag characteristics of a pair of ellipsoids in close proximity," Proceedings of the Institution of Mechanical Engineers, Part M: Journal of Engineering for the Maritime Environment, vol. 216, no. 2, pp. 107-115, 2002.

[24] M. Gevrey, I. Dimopoulos, and S. Lek, "Review and comparison of methods to study the contribution of variables in artificial neural network models," Ecological Modelling, vol. 160, no. 3, pp. 249-264, 2003.

[25] C. Yang, Z. Li, R. Cui, and B. Xu, "Neural network-based motion control of an underactuated wheeled inverted pendulum model," IEEE Transactions on Neural Networks and Learning Systems, vol. 25, no. 11, p. 2004, 2014.

[26] W.-H. Hucho, "Aerodynamics of road vehicles: from fluid mechanics to vehicle engineering," Butterworths, vol. 25, no. 1, pp. 485-537, 1998. 


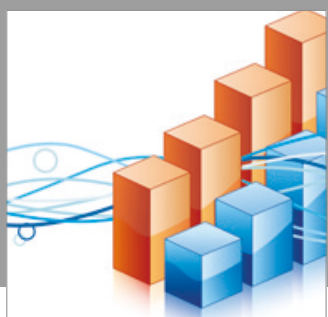

Advances in

Operations Research

vatersals

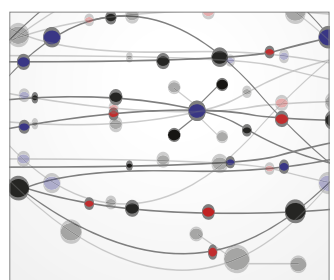

\section{The Scientific} World Journal
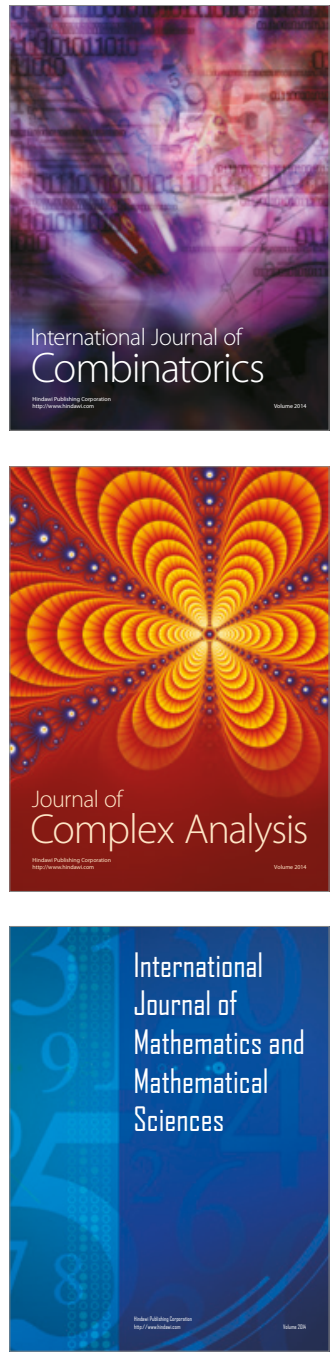
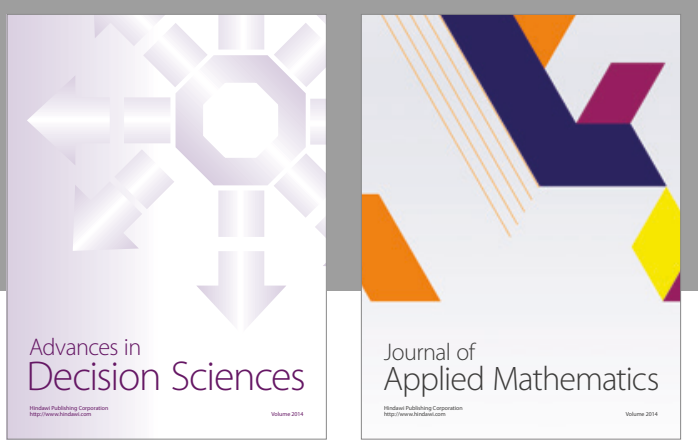

Algebra

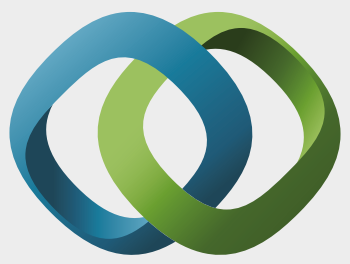

\section{Hindawi}

Submit your manuscripts at

https://www.hindawi.com
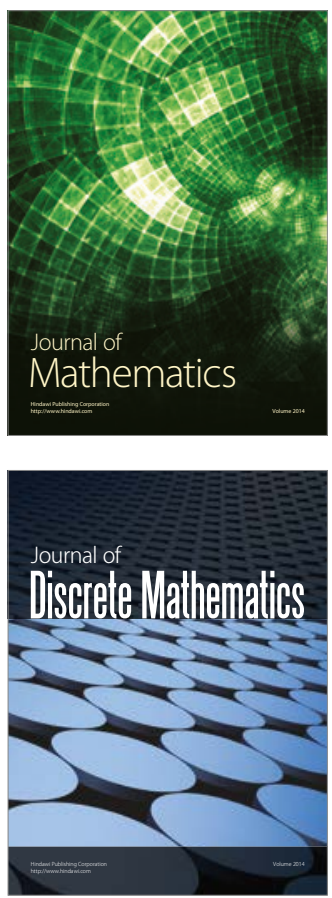

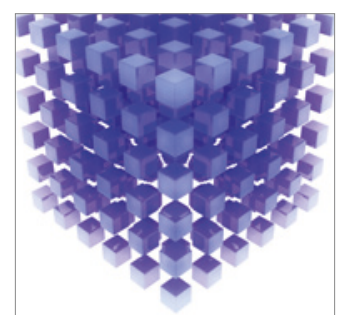

Mathematical Problems in Engineering
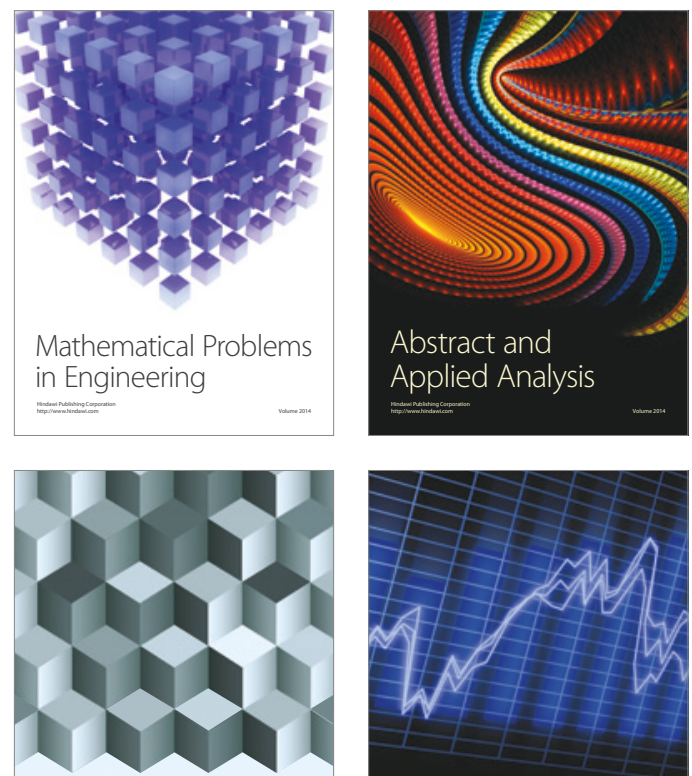

Journal of

Function Spaces

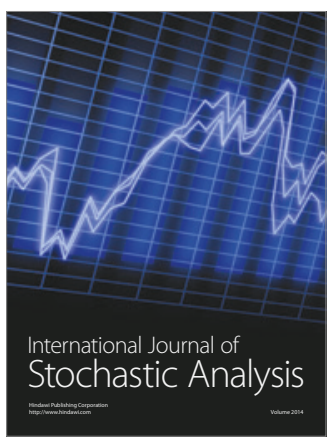

Probability and Statistics
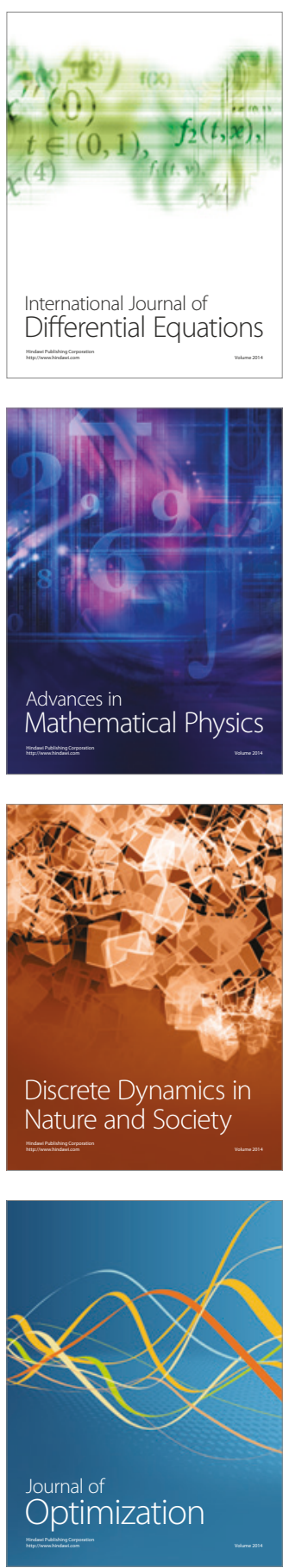